\title{
$n$-Color partition theoretic interpretations of some mock theta functions
}

\author{
A.K. Agarwal* \\ Centre for Advanced Study in Mathematics \\ Panjab University \\ Chandigarh-160014, India \\ E-mail: aka@pu.ac.in
}

Submitted: Mar 13, 2004; Accepted: Sep 14, 2004; Published: Sep 20, 2004

MR Subject Classifications (2000): Primary 05A15, Secondary 05A17, 11 P81.

\begin{abstract}
Using $n$-color partitions we provide new number theoretic interpretations of four mock theta functions of S. Ramanujan.
\end{abstract}

\section{Introduction}

In his last letter to G.H. Hardy, S. Ramanujan listed 17 functions which he called mock theta functions. He separated these 17 functions into three classes. First containing 4 functions of order 3 , second containing 10 functions of order 5 and the third containg 3 functions of order 7. Watson [8] found three more functions of order 3 and two more of order 5 appear in the lost notebook [7]. Mock theta functions of order 6 and 8 have also been studied in [3] and [4], respectively. For the definitions of mock theta functions and their order the reader is referred to [6]. The object of this paper is to provide new number theoretic interpretations of the following mock theta functions:

$$
\begin{gathered}
\Psi(q)=\sum_{m=1}^{\infty} \frac{q^{m^{2}}}{\left(q ; q^{2}\right)_{m}}, \\
F_{0}(q)=\sum_{m=0}^{\infty} \frac{q^{2 m^{2}}}{\left(q ; q^{2}\right)_{m}}, \\
\Phi_{0}(q)=\sum_{m=0}^{\infty} q^{m^{2}}\left(-q ; q^{2}\right)_{m},
\end{gathered}
$$

\footnotetext{
*Supported by CSIR Research Grant No. 25(0128)/02/EMR-I
} 
and

$$
\Phi_{1}(q)=\sum_{m=0}^{\infty} q^{(m+1)^{2}}\left(-q ; q^{2}\right)_{m}
$$

where

$$
(a ; q)_{n}=\prod_{i=0}^{\infty} \frac{\left(1-a q^{i}\right)}{\left(1-a q^{n+i}\right)}
$$

for any constant a.

We remark that $\Psi(q)$ is of order 3 while the remaining three are of order 5 .

Number theoretic interpretations of some of the mock theta functions are found in the literature. For example, $\Psi(q)$ has been interpreted as generating function for partitions into odd parts without gaps [5]. We in this paper use $n$-color partitions (also called partitions with $n$ copies of $n$ and studied first by Agarwal and Andrews in [2]) to give new number theoretic interpretations of the mock theta functions defined above by (1.1)-(1.4). Before we state our main results we recall some definitions from [2].

Definition 1.1. An $n$-color partition (also called a partition with ' $n$ copies of $n$ ') of a positive integer $\nu$ is a partition in which a part of size $n$ can come in $n$ different colors denoted by subscripts: $n_{1}, n_{2}, \ldots, n_{n}$ and the parts satisfy the order $1_{1}<2_{1}<2_{2}<3_{1}<$ $3_{2}<3_{3}<4_{1}<4_{2}<4_{3}<4_{4}<\ldots$. Thus, for example, the $n$-color partitions of 3 are

$$
3_{1}, 3_{2}, 3_{3}, 2_{1} 1_{1}, 2_{2} 1_{1}, 1_{1} 1_{1} 1_{1} \text {. }
$$

Definition 1.2. The weighted difference of two parts $m_{i}, n_{j}, m \geq n$ is defined by $m-n-i-j$ and denoted by $\left(\left(m_{i}-n_{j}\right)\right)$.

We shall prove that the mock theta functions defined by (1.1)-(1.4) have, respectively, the following number theoretic interpretations:

Theorem 1. For $\nu \geq 1$, let $A_{1}(\nu)$ denote the number of $n$-color partitions of $\nu$ such that even parts appear with even subscripts and odd with odd, for some $k, k_{k}$ is a part, and the weighted difference of any two consecutive parts is 0 . Then,

$$
\sum_{\nu=1}^{\infty} A_{1}(\nu) q^{\nu}=\Psi(q)
$$

Example. $A_{1}(8)=3$. The relevant $n$-color partitions are $8_{8}, 7_{5}+1_{1}, 6_{2}+2_{2}$.

Theorem 2. For $\nu \geq 0$, let $A_{2}(\nu)$ denote the number of $n$-color partitions of $\nu$ such that even parts appear with even subscripts and odd with odd greater than 1 , for some $k, k_{k}$ is a part, and the weighted difference of any two consecutive parts is 0 . Then,

$$
\sum_{\nu=0}^{\infty} A_{2}(\nu) q^{\nu}=F_{0}(q)
$$


Theorem 3. For $\nu \geq 0$, let $A_{3}(\nu)$ denote the number of $n$-color partitions of $\nu$ such that only the first copy of the odd parts and the second copy of the even parts are used, that is, the parts are of the type $(2 k-1)_{1}$ or $(2 k)_{2}$, the minimum part is $1_{1}$ or $2_{2}$, and the weighted difference of any two consecutive parts is 0 . Then,

$$
\sum_{\nu=0}^{\infty} A_{3}(\nu) q^{\nu}=\Phi_{0}(q)
$$

Theorem 4. For $\nu \geq 1$, let $A_{4}(\nu)$ denote the number of $n$-color partitions of $\nu$ such that only the first copy of the odd parts and the second copy of the even parts are used, the minimum part is $1_{1}$, and the weighted difference of any two consecutive parts is 0 . Then,

$$
\sum_{\nu=1}^{\infty} A_{4}(\nu)=\Phi_{1}(q)
$$

Remark. We remark that there are $160 n$-color partitions of 8 but only one partition viz., $6_{2}+2_{2}$ is relevant for Theorem 3 and none is relevant for Theorem 4 . Out of 859 $n$-color partitions of 11, none is relevant for Theorems 3-4. Among $18334 n$-color partitions of 17 only two viz., $9_{1}+6_{2}+2_{2}$ and $8_{2}+5_{1}+3_{1}+1_{1}$ satisfy the conditions of Theorem 3 , whereas the lone partition $8_{2}+5_{1}+3_{1}+1_{1}$ satisfies the conditions of Theoem 4 .

Following the method of [1], we give in our next section the detail proof of Theorem 1 and the shortest possible proofs for the remaining theorems. In the sequel $A_{i}(m, \nu),(1 \leq$ $i \leq 4)$, will denote the number of partitions of $\nu$ enumerated by $A_{i}(\nu)$ into $m$ parts, and we shall write

$$
f_{i}(z, q)=\sum_{\nu=0}^{\infty} \sum_{m=0}^{\infty} A_{i}(m, \nu) z^{m} q^{\nu} .
$$

In our last section we illustrate how our new results can be used to yield new combinatorial identities.

\section{Proofs}

Proof of Theorem 1 . We split the partitions enumerated by $A_{1}(m, \nu)$ into two classes: (1) those that contain $1_{1}$ as a part, and those that contain $k_{k},(k>1)$ as a part. Following the method of [1] it can be easily proved that the partitions in Class (1) are enumerated by $A_{1}(m-1, \nu-2 m+1)$ and in Class (2) by $A_{1}(m, \nu-2 m+1)$, and so

$$
A_{1}(m, \nu)=A_{1}(m-1, \nu-2 m+1)+A_{1}(m, \nu-2 m+1) .
$$

From (1.9), we have

$$
f_{1}(z, q)=\sum_{\nu=0}^{\infty} \sum_{m=0}^{\infty} A_{1}(m, \nu) z^{m} q^{\nu} .
$$

Substituting for $A_{1}(m, \nu)$ from (2.1) in (2.2) and then simplifying we get

$$
f_{1}(z, q)=z q f_{1}\left(z q^{2}, q\right)+q^{-1} f_{1}\left(z q^{2}, q\right) .
$$


Setting $f_{1}(z, q)=\sum_{n=0}^{\infty} \alpha_{n}(q) z^{n}$, and then comparing the cofficients of $z^{n}$ on each side of (2.3), we see that

$$
\alpha_{n}(q)=\frac{q^{2 n-1}}{1-q^{2 n-1}} \alpha_{n-1}(q) .
$$

Iterating (2.4) $n$ times and observing that $\alpha_{0}(q)=1$, we find that

$$
\alpha_{n}(q)=\frac{q^{n^{2}}}{\left(q ; q^{2}\right)_{n}} .
$$

Therefore

$$
f_{1}(z, q)=\sum_{n=0}^{\infty} \frac{q^{n^{2}} z^{n}}{\left(q ; q^{2}\right)_{n}} .
$$

Now

$$
\begin{aligned}
\sum_{\nu=0}^{\infty} A_{1}(\nu) q^{\nu} & =\sum_{\nu=0}^{\infty}\left(\sum_{m=0}^{\infty} A_{1}(m, \nu)\right) q^{\nu} \\
& =f_{1}(1, q) \\
& =\sum_{n=0}^{\infty} \frac{q^{n^{2}}}{\left(q ; q^{2}\right)_{n}} \\
& =\Psi(q) .
\end{aligned}
$$

This completes the proof of Theorem 1.

Proof of Theorem 2.

The proof is similar to that of Theorem 1, hence we omit the details and give only the $q$-functional equation used in this case.

$$
f_{2}(z, q)=z q^{2} f_{2}\left(z q^{4}, q\right)+q^{-1} f_{2}(z q, q)
$$

Proof of Theorem 3.

We split the partitions enumerated by $A_{3}(m, \nu)$ into two classes:(1) those that contain $1_{1}$ as a part, and (2) those that contain $2_{2}$ as a part. By using the usual technique we see that the partitions in Class (1) are enumerated by $A_{3}(m-1, \nu-2 m+1)$ and in Class (2) by $A_{3}(m-1, \nu-4 m+2)$. This leads to the identity

$$
A_{3}(m, \nu)=A_{3}(m-1, \nu-2 m+1)+A_{3}(m-1, \nu-4 m+2) .
$$

Using (2.8) one can easily obtain the following $q$-functional equation

$$
f_{3}(z, q)=z q f_{3}\left(z q^{2}, q\right)+z q^{2} f_{3}\left(z q^{4}, q\right) .
$$


Setting $f_{3}(z, q)=\sum_{n=0}^{\infty} \beta_{n}(q) z^{n}$, and noting that $f_{3}(0, q)=1$, we can easily check by coefficient comparison in (2.9) that

$$
\beta_{n}(q)=q^{n^{2}}\left(-q ; q^{2}\right)_{n}
$$

Therefore,

$$
f_{3}(z, q)=\sum_{n=0}^{\infty} q^{n^{2}}\left(-q ; q^{2}\right)_{n} z^{n}
$$

Now

$$
\begin{aligned}
\sum_{\nu=0}^{\infty} A_{3}(\nu) q^{\nu} & =\sum_{\nu=0}^{\infty}\left(\sum_{m=0}^{\infty} A_{3}(m, \nu)\right) q^{\nu} \\
& =f_{3}(1, q) \\
& =\sum_{n=0}^{\infty} q^{n^{2}}\left(-q ; q^{2}\right)_{n} \\
& =\Phi_{0}(q) .
\end{aligned}
$$

This proves Theorem 3 .

Proof of Theorem 4.

The partitions enumerated by $A_{4}(m, \nu)$ are precisely those partitions which belong to Class 1 of the previous case. Therefore,

$$
A_{4}(z, \nu)=A_{3}(m-1, \nu-2 m+1) .
$$

Using Equations (2.8) and (2.12), one can easily obtain the following $q$-functional equation:

$$
f_{4}(z, q)=f_{3}(z, q)-z q^{2} f_{3}\left(z q^{4}, q\right) .
$$

Setting $f_{4}(z, q)=\sum_{n=0}^{\infty} \gamma_{n}(q) z^{n}$, and then comparing the coefficients of $z^{n}$ on each side of (2.13), we see that

$$
\begin{aligned}
\gamma_{n}(q) & =\beta_{n}(q)-\beta_{n-1}(q) q^{4 n-2} \\
& =q^{n^{2}}\left(-q ; q^{2}\right)_{n-1} .
\end{aligned}
$$

This implies that

$$
f_{4}(z, q)=\sum_{n=1}^{\infty} q^{n^{2}}\left(-q ; q^{2}\right)_{n-1} z^{n}
$$


Now

$$
\begin{aligned}
\sum_{\nu=0}^{\infty} A_{4}(\nu) q^{\nu} & =\sum_{\nu=0}^{\infty}\left(\sum_{m=0}^{\infty} A_{4}(m, \nu)\right) q^{\nu} \\
& =f_{4}(1, q) \\
& =\sum_{n=1}^{\infty} q^{n^{2}}\left(-q ; q^{2}\right)_{n-1} \\
& =\sum_{n=0}^{\infty} q^{(n+1)^{2}}\left(-q ; q^{2}\right)_{n} \\
& =\Phi_{1}(q) .
\end{aligned}
$$

This completes the proof of Theorem 4.

\section{New combinatorial identities}

Our Theorems 1-4 can be combined with the known number theoretic interpretations of (1.1)-(1.4) to yield new combinatorial identities. For example, Theorem 1 in view of the known partition theoretic interpretation of $\Psi(q)$ given above in Section 1 gives the

following result:

Theorem 5. For $\nu \geq 1$, the number of $n$-color partitions of $\nu$ such that even parts appear with even subscripts and odd with odd, for some $k, k_{k}$ is a part, and the weighted difference of any two consecutive parts is 0 equals the number of ordinary partitions of $\nu$ into odd parts without gaps.

\section{References}

1. A.K. Agarwal, Rogers-Ramanujan identities for $n$-color partitions, J. Number Theory 28 (1988), 299-305.

2. A.K. Agarwal and G.E. Andrews, Rogers-Ramanujan identities for partitions with " $N$ copies of $N$ ", J. Combin. Theory Ser.A 45, (1987), 40-49.

3. G.E. Andrews and D. Hickerson, Ramanujan's "Lost" Notebook VII: The sixth order mock theta functions, Adv. Math., 89 (1991), 60-105.

4. B. Gordon and R.J. McIntosh, Some eight order mock theta functions, J. London Math. Soc.(2) 62 (2000), 321-335.

5. N.J. Fine, Basic Hypergeometric Series and Applications, Mathematical Surveys and Monographs, No. 27, AMS, (1988)

6. G.H. Hardy and E.M. Wright, An Introduction to the Theory of Numbers, Fifth Edition, Oxford University Press (1978).

7. S. Ramanujan, The Lost Notebook and other Unpublished Papers, Narosa Publishing House, New Delhi, 1988

8. G.N. Watson, The final problem: an account of the mock theta functions, J. London Math. Soc., 11 (1936), 55-80. 